\title{
Emerging photonic devices based on photonic liquid crystal fibers
}

\author{
T. R. Woliński, ${ }^{1 *}$ S. Ertman, ${ }^{1}$ D. Budaszewski, ${ }^{1}$ M. Chychłowski, ${ }^{1}$ A. Czapla, ${ }^{1}$ \\ R. Dąbrowski, ${ }^{2}$ A. W. Domański, ${ }^{1}$ P. Mergo, ${ }^{3}$ E. Nowinowski-Kruszelnicki, ${ }^{2}$ \\ K. A. Rutkowska, ${ }^{1}$ M. Sierakowski, ${ }^{1}$ M. Tefelska ${ }^{1}$ \\ ${ }^{I}$ Faculty of Physics, Warsaw University of Technology, Koszykowa 75, 00-662 Warszawa,Poland \\ ${ }^{2}$ Military University of Technology, Warszawa, ${ }^{3}$ Maria Curie-Skłodowska University, Lublin, Poland
}

Received March 25, 2011; accepted March 27, 2011; published March 31, 2011

\begin{abstract}
Photonic liquid crystal fibers (PLCFs) define a new class of optical waveguides that combines unique properties of microstructured photonic crystal fibers and liquid crystals. Current progress of research in the field of PLCFs means that a new class of emerging photonics devices can be expected, i.e. tunable attenuators, tunable broad-band filters, tunable polarizers (with an arbitrary PDL and a variable axis of polarization), tunable waveplates, and tunable phase shifters with high retardance will appear in the near future. In this work we would like to discuss this perspective, paying special attention to two most challenging technological issues such as control of LC molecules orientation and permanent connecting of PLCFs to standard fibers.
\end{abstract}

Transmission properties of photonic crystal fibers (PCFs) can be tailored in a wide range by changing geometry of their microstructured cladding [1]. Generally, in the PCFs light can be guided by two different mechanisms: indexguiding (similar to the classical waveguide effect based on mTIR- modified total internal reflection) and the photonic band-gap (PBG) effect. The PBG propagation occurs when the effective refractive index of the microstructured cladding is higher than the refractive index of the core, and in this case only selected wavelengths can be guided [1]. Transmission properties of PCFs can be also arbitrarily changed by filling their micro-holes with various substances [2] and liquid crystals (LCs) belong to the most interesting materials for these applications due to the high sensitivities to external physical fields. The first paper on photonic liquid crystal fibers (PLCFs) has been published in 2003 [3]. Since that time there has been a continuous increase in the number of research groups working in the field, and thermal tuning of the photonic band-gap in the PLCFs has been shortly reported also by different authors [4-6]

Electrical tuning in a hollow-core liquid-crystal photonic crystal fiber was demonstrated in 2004 [7] and also in silica glass-core photonic liquid crystals fibers [89]. In Ref. [9] thermal tuning of the PLCF guiding mechanism was presented by using a special lowbirefringence LC mixture with the ordinary refractive index lower (in a certain temperature range) than the refractive index of the silica glass. The efficiency of electrical tuning could be significantly improved by using dual-frequency liquid crystals [10-11]. Also, all-optical tuning was demonstrated in a PCF filled with a LC doped

http://www.photonics.pl/PLP either with dyes [12] or with azobenzene [13]. Moreover, frequency tunability of solid-core photonic crystal fibers filled with nanoparticle-doped liquid crystals has been reported [14] recently.

One of the most interesting features of the PLCFs is the possibility of dynamic tuning of their polarization properties due to reorientation of LC molecules induced by external fields. Tunable polarization dependent losses [15], tunable single polarization operation [16] and tunable birefringence [11, 17-19] have been obtained in silica-based PCFs filled with LCs. Other interesting effects observed in the LC-PCFs were based on a PCF made of multi-component glasses, with increased value of the refractive index, so that index-guiding propagation was possible even after infiltration with LCs. In particular, low-loss propagation and continuously tunable birefringence was experimentally demonstrated [20].

Recently, a number of prototype tunable devices based on PLCFs have been developed in our laboratory [21, 22]. These devices include tunable attenuators, tunable broadband filters, tunable polarizers (with arbitrary polarization dependent losses PDL and variable axis of polarization), tunable waveplates, and tunable phase shifters with high retardance. Such components can be further utilized for assembling more sophisticated devices such as all-in-fiber polarization controllers and polarization mode dispersion compensators.

However, characterization of the prototype devices has led us to the conclusion that our current PLCF technology still needs to be improved, since the achieved repeatability of measurement results has not been sufficient in view of potential practical applications, where high stability of measured parameters is required. In particular, two important manufacturing steps should be further optimized. The first issue is controlling the orientation and ordering of the LC molecules infiltrating the PCF micro-holes. Another essential issue is permanently connecting PLCFs with standard input/output fibers. The letter presents recent progress in these two areas and discusses some future perspectives.

In our early experiments, microstructured fibers were infiltrated with LCs by the capillary action just by immersing one end of the PCF in a container with an LC 
for a few minutes. As a result, LC molecules orientation within the fiber holes was not controlled in any way, and might differ, depending on the pressure and time of infiltration. However, techniques for the controlled process of LC infiltration have been developed in the last few years [23-25]. Our approach is based on creation of a thin aligning film of a polymer inside each micro-hole. To obtain a thin layer, we fill the fiber with a dissolved polymer then the solution is pulled out of the fiber by leaving the thin film on the holes. In next step, the fiber is heated for a while to evaporate the remains of the solvent. The last step depends on the type of the polymer used for aligning - thermal polymerization may be obtained by baking the sample, and photo-polymerization can be induced by irradiating it with ultra-violet (UV) light [2325]. In practice, thermal polymerization is easier, so thermal-alignment is more repeatable than photoalignement, where careful and precise irradiation of the PCF is required. However, in the case of thermal polymerization only one type of anchoring conditions is possible, so to obtain a different orientation of the LC molecules it is necessary to use different types of polymer. On the contrary, photo-aligning is much more flexible, since the direction of the orientation of the LC molecules can be defined by the polarization of the UV light used to induce a photo-chemical reaction. For example, it allows to obtain a few different orientations within one fiber. An example of such a sample is shown in Fig.1. Two different orientations were obtained by selective irradiation of a capillary with polarized UV light. As could be noticed in Fig. 1, we have already obtained quite good results in the field of the LC alignment inside cylindrical micro-holes.
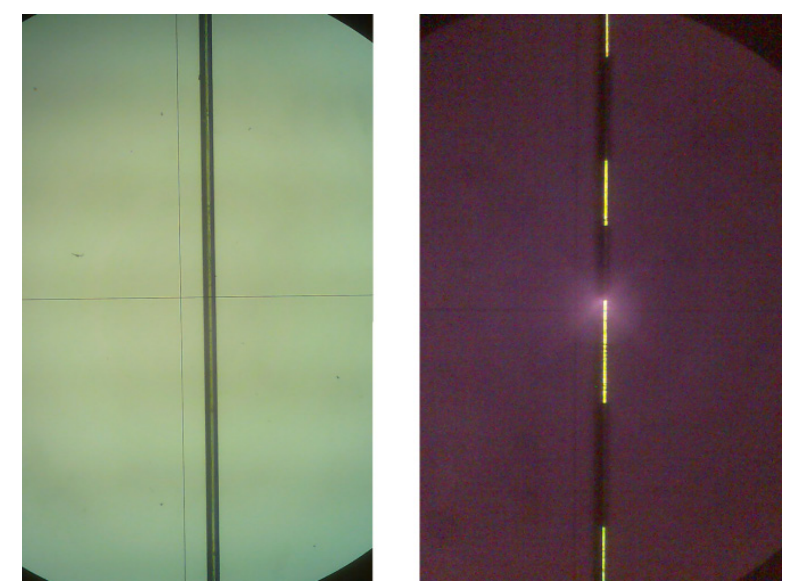

Fig. 1. A capillary filled with LC observed in a polarizing light microscope - polarizers parallel (left) and crossed (right). The sample was selectively irradiated with polarized UV light and thus two different orientations can be clearly distinguished.
To ensure high repeatability of results, a further research effort in the field should be continued. It may occur that for simpler devices, where only one orientation is needed, the thermal alignment may be the optimal technological choice.

Another area of our technological research activities is the creation of permanent connections between PLCFs and standard fibers. In many applications a standard single mode fiber (SMF) should be connected to both ends of the PLCF in order to obtain a device that can be used, e.g. in telecommunication systems. Direct splicing is not possible because a high temperature (in both fusion and filament splicers) may permanently destroy the LC. Luckily, it is possible to "push" the LC deep into the PCF (with high pressure air), so both ends of the host fiber can be accessible for splicing, using the same techniques like described in [26].

However, some very useful properties have been obtained in PLCFs based on the multi-component glasses with increased value of the refractive index, allowing for broadband index guiding propagation. In most cases, splicing the soft multi-component glasses with silica glass is a very challenging task since chemical compositions and thermal properties (especially melting temperatures) of both glasses are totally different. Sometimes the difference in their thermo-chemical properties is so significant that splicing becomes almost impossible. Thus some alternative methods of effective fiber connections should be developed. One of the ideas used in our research is a so-called "precise capillary connection", where the fibers to be connected are placed inside the capillary, and then secured with epoxy glue. As an extension of this method we may consider "hot capillary connections", the technique which exploits thermal collapse of the capillary made of "soft" glass with the melting temperature lower than the connected fibers. In this case we don't have to worry about adjusting the fibers, since the collapsing capillary will "automatically" center both fibers.

The last alternative method is gluing with UV curable adhesives. We are currently testing this method since the initial results are very promising. There is a broad choice of UV-curable adhesives that are transparent for optical wavelengths and it is also possible to choose the adhesive of the refractive index close to the refractive indices of the connected fibers. The main advantage of UV-curable gluing is that the fibers can be precisely adjusted to optimize coupling, and then the curing light can be switched on. An example of an electrically tunable device based on the PLCF with UV-curable connections is presented in Fig. 2. A micrograph of one connection is also presented in Fig. $2 \mathrm{~b}$. 


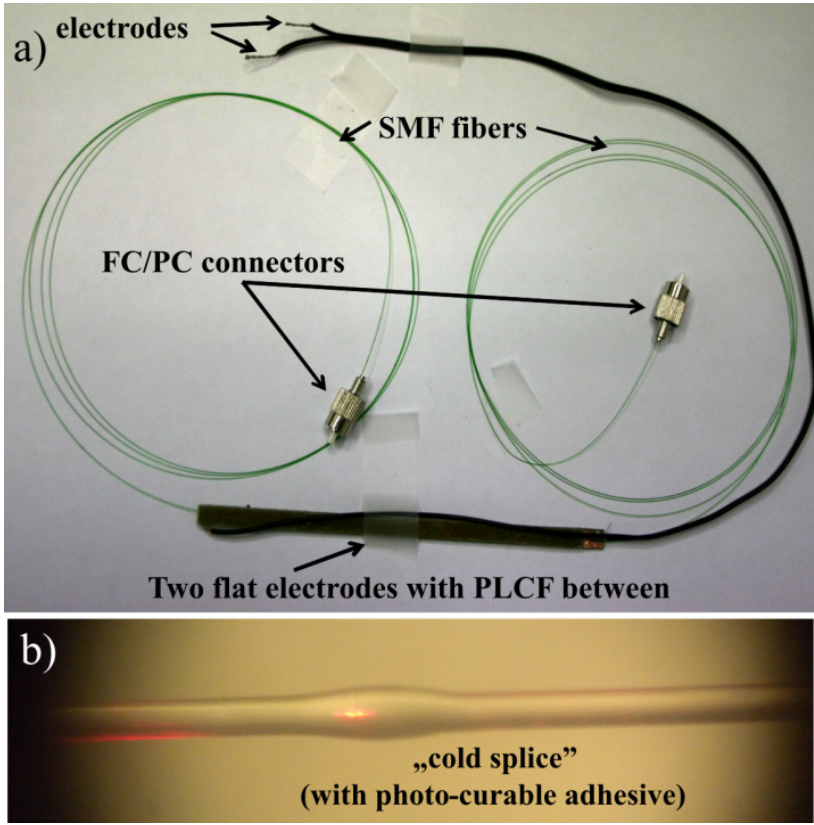

Fig. 2. Prototype of the electrically tunable phase shifter based on a high index core PLCF: a) general overview of the device; b) close up to the "cold splice" made with a photo-curable adhesive.

Photonic Liquid Crystal Fibers have become over the last seven years an emerging, but still not matured technology. However, a number of novel photonic devices based on the PLCFs have been demonstrated and developed at the Warsaw University of Technology in collaboration with other research groups in Poland and worldwide. A special selection of the quest liquid crystalline materials and host photonic crystal fibers, as well as the possibility of simple adjustment and control of their parameters can significantly broaden up a list of their potential applications. Current works involve basic and applied research leading to new applications of the LC PCF-based devices. Special attention is devoted to technological procedures that will ensure the highest possible level of repeatability, so it could be expected that in the near future first devices based on the PLCFs can find useful practical applications.
The authors acknowledge the Foundation for Polish Science (Mistrz Programme) and also a fruitful collaboration with Mr. D. Pysz (ITME, Poland), Dr. R. Buczyński (Warsaw Univ., Poland), Prof. W. Bock (UQO, Canada), Prof. J. Oton (Madrid, Spain), Prof. K. Neyts (Ghent, Belgium), Prof. P. Shum, and Dr. Juan Juan D. Hu (NTU, Singapore) in the field liquid crystal photonic crystal fibers. This work was also partially supported by the Polish Ministry of Science and Education under the grants N517 554139 and N507 439539.

\section{References}

[1] P.J. Russell, J. Lightwave Technol. 24, 4729 (2006).

[2] D.B. Eggleton et al., Opt. Exp. 9, 698 (2001).

[3] T.T. Larsen et al., Opt. Exp. 11, 2589 (2003).

[4] T.R. Wolinski et al., Opto-Electronics Rev. 13(2), 177 (2005).

[5] J. Du et al., Appl. Opt. 47, 5321 (2008).

[6] M.Y. Jeon, J.H. Kim, Japan. Jour. Appl. Phys. 47(4), 2174 (2008).

[7] Q. Lu, S.T.Wu, Appl. Phys. Lett. 85, 2181 (2005).

[8] M.W. Haakestad et al., IEEE Photon. Technol. Lett. 17, 819 (2005).

[9] T.R. Wolinski et al., Meas. Sci. Technol. 17, 985 (2006).

[10] L. Scolari et al., Opt. Expr. 13, 7483 (2005).

[11] A. Lorenz et al., Opt. Expr. 16, 19375 (2008).

[12] T. Alkeskjold et al., Opt. Expr. 12, 5857 (2004).

[13] V.K. Hsiao, C.-Y. Ko, Opt. Expr. 16, 12670 (2008)

[14] L. Scolari et al., Opt. Expr. 17, 3754 (2009).

[15] T.T. Alkeskjold, A. Bjarklev, Opt. Lett. 32, 1707 (2007).

[16] T.R. Wolinski, Meas. Sci. Technol. 18, 3061 (2007).

[17] T.R. Woliński et al., Opt. Quantum Electron. 39(12-13), 1021 (2007).

[18] S. Ertman et al., Opto-Electronics Review 17, 150 (2009).

[19] L. Wei et al., Appl. Opt. 48, 497 (2009).

[20] S. Ertman et al., Opt. Expr. 17, 19298 (2009).

[21] T.R. Woliński et al., 2010 International Conference on Photonics, ICP2010, art. no. 5604369.

[22] T.R. Woliński et al., Proc. SPIE 7955, 795502 (2011).

[23] V.V. Presnyakov et al., Proc. SPIE 6017, 60170J-1-7 (2005).

[24] S. Ertman et. al., Proc. SPIE 6587, 658706 (2007).

[25] M.S. Chychłowski et al., Acta Phys. Polonica A 118, 1100 (2010).

[26] M. Murawski et al., Phot. Lett. Poland 1(3) 115 (2009). 